\title{
Comparative Study on Two Decoupling Control Methods of Doubly-fed Induction Generator
}

\author{
Xianghong $\mathrm{Xu}^{\mathrm{a}} \quad$ Ziqiang $\mathrm{Lu}^{\mathrm{b}}$ \\ Shazhou Professional Institute of Technology, Zhangjiagang 215600, China \\ aemail: xxh602@126.com, bemail:2538561733@qq.com
}

\begin{abstract}
Keywords: Doubly-fed induction generator, vector control, Internal Model Control, decoupling control.

Abstract. Doubly-fed induction generator is a multivariable, strong-coupling, nonlinear controlled object. The paper makes a comparative study on two decoupling control methods based on vector control and Internal Model Control theory for decoupling voltages of DFIG. Theoretical analysis and simulation results show that the designed controllers based on IMC principle have better performance than of vector control.
\end{abstract}

\section{Introduction}

Doubly-fed induction generator (DFIG) structure is simple, solid and reliable, which is the core part of wind power generation system. The running status of the control system, on the wind of the whole system performance, efficiency and output power quality has important influence. But the doubly-fed generator is a multivariable, nonlinear and strong coupling control object. As is known to all, under the three-phase static coordinate system, there are some parameters in the mathematical model of doubly-fed generator changes over on the rotor Angle and rotational speed, the dynamic mathematical model of doubly-fed generator complex, analysis and solve the difficulties. In order to get the ideal control performance, the key lies in the decoupling[1]. Traditional vector control is the use of coordinate transformation method, the equivalent for dc motor, induction motor has realized the motor excitation current component and torque component of decoupling, the decoupling method is a kind of more successful[2]. But still exist after the vector transformation $\mathrm{M}$ axis and $\mathrm{T}$ axis of cross coupling between electric potential, must be decoupled. In this paper, we will to decouple the doubly-fed generator cross coupling potential, to take on the basis of the traditional vector control feedback decoupling method and based on internal model decoupling method of two decoupling control strategy theory and the simulation contrast research. From the simulation of decoupling effect, internal model decoupling control scheme, make the output signals can be quickly and accurately track the input signal, its performance is superior to the feedback decoupling control scheme[3].

\section{Theoretical analysis}

\section{Cross coupling voltage feedback decoupling control}

With stator current and stator flux linkage as state variables, the equation of state of doubly-fed generator is as follows:

$$
\left[\begin{array}{l}
U_{s} \\
U_{r}
\end{array}\right]=\left[\begin{array}{cc}
-R_{s} & j \omega_{g} \\
\frac{L_{s} R_{r}}{L_{m}}+j \omega_{s} \frac{\sigma L_{s} L_{r}}{L_{m}} & \frac{R_{r}}{L_{m}}+j \omega_{s} \frac{L_{r}}{L_{m}}
\end{array}\right]\left[\begin{array}{l}
I_{s} \\
\Psi_{s}
\end{array}\right]+\left[\begin{array}{cc}
0 & 1 \\
\frac{\sigma L_{s} L_{r}}{L_{m}} & \frac{L_{r}}{L_{m}}
\end{array}\right]\left[\begin{array}{l}
p I_{s} \\
p \psi_{s}
\end{array}\right]
$$

Rotor stator flux voltage equation can be obtained: 


$$
\left\{\begin{array}{l}
u_{M 2}=\left(\frac{R_{r} L_{s}}{L_{m}}+\frac{\sigma L_{s} L_{r}}{L_{m}} p\right) i_{M 1}-\frac{\omega_{s} \sigma L_{s} L_{r}}{L_{m}} i_{T 1}+\frac{R_{r}}{L_{m}} \psi_{s} \\
u_{T 2}=\frac{\omega_{s} \sigma L_{s} L_{r}}{L_{m}} i_{M 1}+\left(\frac{R_{r} L_{s}}{L_{m}}+\frac{\sigma L_{s} L_{r}}{L_{m}} p\right) i_{T 1}+\frac{\omega_{s} L_{r}}{L_{m}} \psi_{s}
\end{array}\right.
$$

Set: $\quad u_{M 2}-\frac{R_{r}}{L_{m}} \Psi_{s}=u_{M 2}^{*}, \quad u_{T 2}-\frac{\omega_{s} L_{r}}{L_{m}} \Psi_{s}=u_{T 2}^{*}$

$$
\left\{\begin{array}{l}
u_{M 2}^{*}=\left(\frac{R_{r} L_{s}}{L_{m}}+\frac{\sigma L_{s} L_{r}}{L_{m}} p\right) i_{M 1}-\frac{\omega_{s} \sigma L_{s} L_{r}}{L_{m}} i_{T 1} \\
u_{T 2}^{*}=\frac{\omega_{s} \sigma L_{s} L_{r}}{L_{m}} i_{M 1}+\left(\frac{R_{r} L_{s}}{L_{m}}+\frac{\sigma L_{s} L_{r}}{L_{m}} p\right) i_{T 1}
\end{array}\right.
$$

Traditional feed forward decoupling is the input voltage of the generator $u_{M 2}$ and $u_{T 2}$ the additional decoupling $-\hat{\sigma} \hat{L}_{s} \hat{L}_{r} \omega_{s} i_{M 1}^{*} / \hat{L}_{m}$ and $\sigma \hat{L}_{s} \hat{L}_{r} \omega_{s} i_{T 1}^{*} / \hat{L}_{m}$, to offset the coupling effect between excitation current and torque, decoupling of electric current is given current $i_{M 1}^{*}$ and $i_{T 1}^{*}$. And generator excitation, the coupling effect between the torque current is, in fact, the actual current generator, in order to achieve better decoupling effect, given the current value should be actual values instead of using electricity, this is the feedback decoupling control method, the control structure is shown in Fig.1.

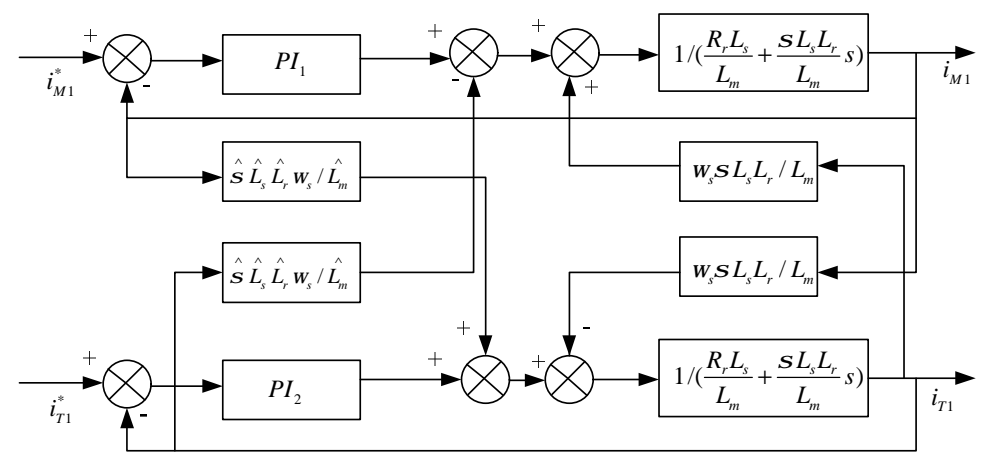

Fig.1 Feedback decoupling control principle diagram

Fig. 1 estimates the box $\sigma L_{s} L_{r} \omega_{s} / L_{m}$ used for decoupling $P I_{1}$ and regulator $P I_{2}$, as the core of the two control closed loop of the stator current component. Feedback decoupling control is based on stator current feedback without delay and cross coupling the quantity of estimates with actual value on the basis of perfectly. However, due to the filter and transformation of the stator current feedback is introduced into link delay, will cause the actual current and the deviation of calculated value; In addition, the deviation of the parameter estimates will also make the feedback decoupling control effect. So feedback decoupling control is sensitive to parameter variation, decoupling effect depends on the accurate mathematical model of controlled object, is unlikely to be completely decoupled.

\section{Internal model decoupling control of cross coupling electric potential}

In order to overcome the decoupling effect is dependent on the accurate mathematical model of the object, must seek some control strategy of model accuracy is not high, intelligent control method such as fuzzy control. But they are generally more complex, engineering implementation is difficult. Internal model control is developed the chemical process control practical very strong control method. Internal model control is not overly dependent on the mathematical model of controlled object, low accuracy of the model, the system tracking good regulation performance, strong robustness, can eliminate the interference of unpredictable; The designed controller structure is simple, clear direction, with single parameter online calculation convenient, easy to implement in 
engineering, is a kind of advanced control technology.

If you ignore unpredictable disturbance impact on system, commonly used internal model control system structure as shown in Fig.2, in the Fig $G(s)$ as the controlled object, $G(s)$ for the controlled object internal model and internal model controller $C_{I M C}(s)$ for equivalent feedback controller, $C(s)$ for the output of the internal model, $Y_{m}(s)$ for the system output $G(s)$ and $d(s)$ the difference between the internal output model, and input $R(s)$ and output $Y(s)$ of the system.

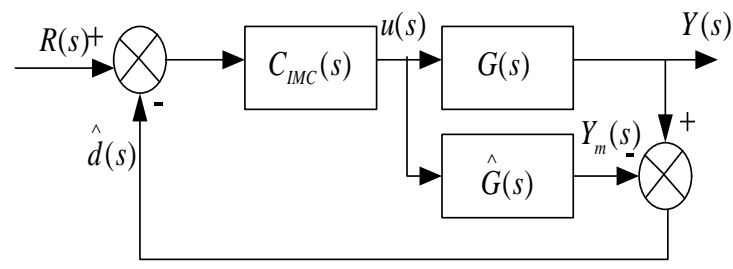

Fig. 2 The relative position between the ball and raceway

Set:

$$
C_{I M C}(s)=\frac{C(s)}{1+\hat{G}(s) C(s)}
$$

If the model and the object mismatch (that is $\hat{G}(s) \neq G(s)$ ), can be used to the output caused by the deviation. If the figure is the before mentioned in Fig.(2) positive feedback on forming the equivalent controller, called the equivalent structure of internal model control, namely:

$$
F(s)=\left[1-C_{I M C}(s) \hat{G}(s)\right]^{-1} C_{I M C}(s)
$$

In the actual design internal model controller, we usually add a low pass filter, by adjusting the structure and parameters to stabilizing system, which adopts of the controller, can make the control system has a certain robustness:

$$
C_{I M S}(s)=\hat{G}^{-1}(s) L(s)
$$

\section{Internal model control design}

In accordance with the doubly-fed generator mathematical model (3) to carry on the design internal model controller, there are:

$$
Y(s)=G(s) U(s)
$$

Parameters as follows:

$$
\begin{gathered}
Y(s)=\left[\begin{array}{ll}
i_{m 1}(s) & i_{T 1}(s)
\end{array}\right]^{T} \\
U(s)=\left[\begin{array}{ll}
u_{M 1}^{*}(s) & u_{T 1}^{*}(s)
\end{array}\right]^{T} \\
G(s)=\left[\begin{array}{ll}
G_{1}(s) & G_{2}(s)
\end{array}\right]^{-1} \\
G_{1}(s)=\left[\begin{array}{ll}
\frac{R_{r} L_{s}}{L_{m}}+\frac{\sigma L_{s} L_{r}}{L_{m}} s & \frac{\omega_{s} \sigma L_{s} L_{r}}{L_{m}}
\end{array}\right]^{T} \\
G_{2}(s)=\left[\begin{array}{ll}
-\frac{\omega_{s} \sigma L_{s} L_{r}}{L_{m}} & \frac{R_{r} L_{s}}{L_{m}}+\frac{\sigma L_{s} L_{r}}{L_{m}} s
\end{array}\right]^{T} \\
L(s)=\frac{\lambda}{s+\lambda} I
\end{gathered}
$$

It is can be obtained:

$$
F(s)=\left[1-C_{I M C}(s) \hat{G}(s)\right]^{-1} C_{I M C}(s)=\frac{\lambda}{s} \hat{G}^{-1}(s)
$$

Internal model decoupling control method adopts control structures shown in figure 3. Internal model decoupling actually compared with feedback decoupling, its decoupling of voltage on electric potential cross coupling calculation is based on the generator of a given current and feedback current deviation. It rejects the feedback decoupling only decouple until after generator 
coupled effects of defects, avoid the feedback decoupling voltage calculation, stator current delay on the calculation of decoupling voltage.

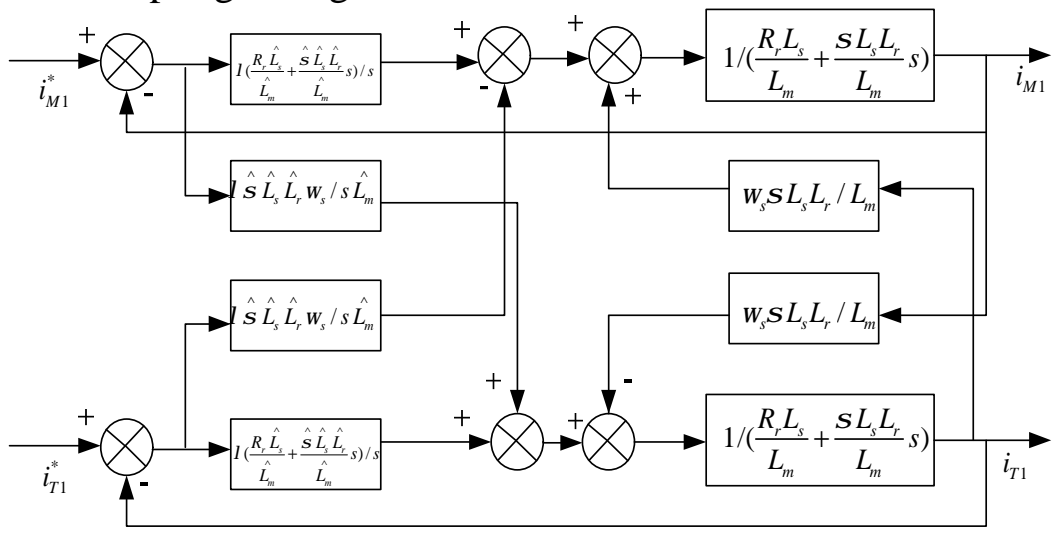

Fig.3 The decoupling internal model control principle diagram.

\section{Result and discussion}

In the doubly-fed generator double PWM in variable frequency power supply and speed under the condition of known, the observer for vector control of doubly-fed generator under observation and identification. Based on Matlab simulation model is established, including:the data of doubly-fed generator power.

The following is based on the Matlab/Simulink simulation results. Figure 8 is active power reference value at $\mathrm{t}=4 \mathrm{~s}$ from $800 \mathrm{w}$ to $1200 \mathrm{w}$, while keeping the reference value of reactive power is constant, independent adjust the active power $\mathrm{P}$ simulation diagram. Figure 9 is the reactive power reference value at $\mathrm{t}=4 \mathrm{~s}$ from $200 \mathrm{w}$ to $0 \mathrm{w}$, at the same time, active power reference value under the condition of unchanged, independent adjust reactive power $\mathrm{Q}$ simulation diagram.

Can be seen from the above theoretical derivation and simulation results, the doubly-fed generator active power and reactive power adjustment process is independent of each other. In power decoupling and internal model controller design, can maintain traceability of flux and the parameters of the good, the active power and reactive power decoupling, fully improve the accuracy and robustness of the control system.

\section{Acknowledgements}

This work was financially supported by Jiangsu university brand professional construction engineering projects in 2015(PPZY2015B184) and College students' innovative training program in Jiangsu province 2016(201611288010Y)

\section{References}

[1]E.Y.Ho and P.Sen. Decoupling Control of Induction Motor Drives. IEEE Trans. IA, Vol.35,No.2,1998.

[2]J.Jung.A. Dynamic Decoupling Control Scheme for High-Speed Operation of induction Motors .IEEE Trans. IE, Vol.46, No.1,1999.

[3] Chen.Y. Based on adaptive internal model control of induction motor. The regulation of the current journal of electrical engineering technology, 2000 (2). 